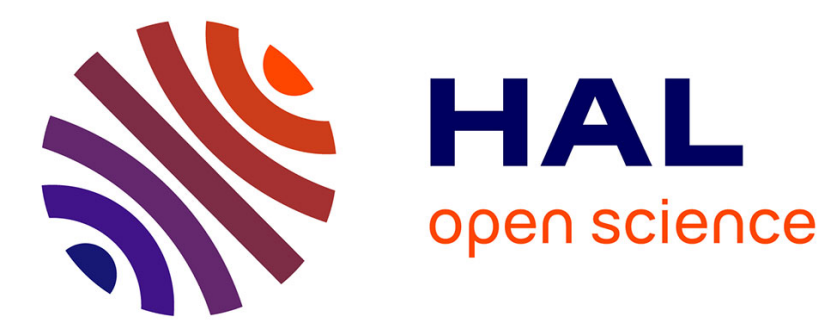

\title{
Twisted absolute instability in lifted flames
}

Joseph W. Nichols, Jean-Marc Chomaz, Peter J. Schmid

\section{To cite this version:}

Joseph W. Nichols, Jean-Marc Chomaz, Peter J. Schmid. Twisted absolute instability in lifted flames. Physics of Fluids, 2009, 21 (1), pp.015110. 10.1063/1.3068758 . hal-01002605

\section{HAL Id: hal-01002605 \\ https://hal-polytechnique.archives-ouvertes.fr/hal-01002605}

Submitted on 7 Jul 2014

HAL is a multi-disciplinary open access archive for the deposit and dissemination of scientific research documents, whether they are published or not. The documents may come from teaching and research institutions in France or abroad, or from public or private research centers.
L'archive ouverte pluridisciplinaire HAL, est destinée au dépôt et à la diffusion de documents scientifiques de niveau recherche, publiés ou non, émanant des établissements d'enseignement et de recherche français ou étrangers, des laboratoires publics ou privés. 


\section{AIP $\mid$ Physics of Fluids}

\section{Twisted absolute instability in lifted flames}

Joseph W. Nichols, Jean-Marc Chomaz, and Peter J. Schmid

Citation: Physics of Fluids (1994-present) 21, 015110 (2009); doi: 10.1063/1.3068758

View online: http://dx.doi.org/10.1063/1.3068758

View Table of Contents: http://scitation.aip.org/content/aip/journal/pof2/21/1?ver=pdfcov

Published by the AIP Publishing

\section{Articles you may be interested in}

Recirculation zone dynamics of a transversely excited swirl flow and flame

Phys. Fluids 24, 075107 (2012); 10.1063/1.4731300

Acoustically perturbed turbulent premixed swirling flames

Phys. Fluids 23, 037101 (2011); 10.1063/1.3553276

A numerical investigation of flame liftoff, stabilization, and blowout

Phys. Fluids 18, 043603 (2006); 10.1063/1.2191851

Coupled hydrodynamic and diffusional-thermal instabilities in flame propagation at subunity Lewis numbers

Phys. Fluids 17, 074106 (2005); 10.1063/1.1964845

The role of secondary instabilities in the stabilization of a nonpremixed lifted jet flame

Phys. Fluids 13, 2662 (2001); 10.1063/1.1386935

\section{A|P| $\left.\right|_{\text {Applied Physics }} ^{\text {Journal of }}$}

Journal of Applied Physics is pleased to announce André Anders as its new Editor-in-Chief 


\title{
Twisted absolute instability in lifted flames
}

\author{
Joseph W. Nichols, ${ }^{\text {a) }}$ Jean-Marc Chomaz, and Peter J. Schmid \\ Laboratoire d'Hydrodynamique (LadHyX), CNRS, École Polytechnique, 91128 Palaiseau cedex, France
}

(Received 21 August 2008; accepted 18 November 2008; published online 29 January 2009)

The theory of resonant modes is extended to finite length systems containing pinch points of complex axial wavenumber $k_{0}$ and frequency $\omega_{0}$ with arbitrary $\omega_{k k}^{0}=\partial^{2} \omega / \partial k^{2}$. The quantity $\omega_{k k}^{0}$ is shown to be an important indicator of how streamwise boundary conditions modify the local absolute mode at $\left(k_{0}, \omega_{0}\right)$. In particular, when $\operatorname{Im}\left(\omega_{k k}^{0}\right)>0$, the pinch point is twisted, and resonant modes owing to streamwise boundary conditions may then have growth rates greater than that of the unbounded absolute mode. In this case, global instability may occur while the flow is only convectively unstable. The premixing zone between the nozzle and a lifted flame on a variable-density jet is an example of a streamwise-confined system containing a twisted pinch point. For this system, linear stability analysis is employed to locate resonant modes along a solution curve in the complex $k$ and $\omega$ planes. The orientation of the solution curve predicts destabilization owing to streamwise confinement as well as increasing global frequency with decreasing lift-off height as observed in previous direct numerical simulations. The theory also suggests that low-frequency fluctuations observed in the simulations may be explained by beating between two resonant modes of slightly differing frequencies. (C) 2009 American Institute of Physics. [DOI: 10.1063/1.3068758]

\section{INTRODUCTION}

When the flow rate of a nonpremixed round fuel jet is sufficiently large, a diffusion flame cannot be sustained in the immediate vicinity of the nozzle. Instead, the flame lifts off of the nozzle and stabilizes a distance $H$ downstream (see Fig. 1) at which point a kinematic balance is achieved between the velocity of the oncoming reactants and the flame speed given by triple flame theory. ${ }^{1,2}$ In a previous study, ${ }^{3}$ numerical simulations of lifted flames on absolutely unstable round fuel jets have shown that the flow is stabilized by decreasing the flame lift-off height $H$ below a critical value $H_{c}$. Before stabilization, the global frequency $\omega_{G}$ of the lifted flame is observed to increase as $H$ decreases. Also, just on the threshold of stabilization, a low-frequency fluctuation is observed to arise in addition to the instability at $\omega_{G}$.

The lifted flame may be considered as a streamwise confinement to the absolutely unstable jet upstream. In this case, the jet is bounded by the nozzle and the sharp acceleration of the flow through the flame base. This acceleration acts as an internal Neumann boundary condition to perturbations in the flow, whereas the perturbations may be assumed to vanish at the inlet, as shown in Fig. 1(b) where $u^{\prime}$ denotes a generalized perturbation. Such boundary conditions are believed to only be stabilizing when applied at upstream and downstream locations. ${ }^{4,5}$ Using a lifted flame with an above critical lift-off height as an example, however, we will show here that streamwise confinement may also be destabilizing, contrary to previous results.

Recently, it has been shown that transverse confinement

${ }^{a)}$ Electronic mail: joseph.nichols@ladhyx.polytechnique.fr. of parallel flows enhances absolute instability for planar jets and wakes, ${ }^{6}$ rotating boundary layers, ${ }^{7}$ and round jets. ${ }^{8}$ Furthermore, transverse confinement may render an otherwise convectively unstable flow globally unstable owing to growth and propagation of disturbances in the cross-stream direction. ${ }^{9,10}$ In this case, growth and propagation are represented by instability modes resembling "leaky" modes ${ }^{11,12}$ which grow exponentially in the transverse direction, and thus were previously thought to be unphysical. In this paper, we suggest that streamwise confinement may have a similar destabilizing role. In the case of streamwise confinement, however, we will show that the destabilization arises from twisted pinch points (i.e., with $\operatorname{Im}\left(\omega_{k k}^{0}\right)>0$ at the pinch point) rather than leaky modes. It is interesting to note, though, that twisted pinch points, like leaky modes, are at first a seemingly paradoxical source of destabilization since they are associated with linearized Ginzburg-Landau (LGL) equations which are noncausal.

Indeed, local stability analysis of a variable-density jet ${ }^{13}$ reveals a pinch point with $\operatorname{Im}\left(\omega_{k k}^{0}\right)>0$, where $k$ and $\omega$ denote axial wavenumber and frequency, respectively, and $\omega_{k k}^{0}$ denotes $\partial^{2} \omega / \partial k^{2}$ evaluated at the pinch point. The condition $\operatorname{Im}\left(\omega_{k k}^{0}\right)>0$, however, is forbidden in the context of the LGL equation studied in Ref. 4, since the problem is then noncausal, as discussed below. The purpose of this paper is to revisit and extend the theory discussed in Refs. 4 and 5 to systems with pinch points with arbitrary $\omega_{k k}^{0}$. In particular, we will see that systems (such as the lifted flame) containing a bounded region of absolute instability with $\operatorname{Im}\left(\omega_{k k}^{0}\right)>0$ may be destabilized by resonant modes with growth rates larger than that of the unbounded absolute mode, i.e., they may be globally unstable while locally convectively unstable. 


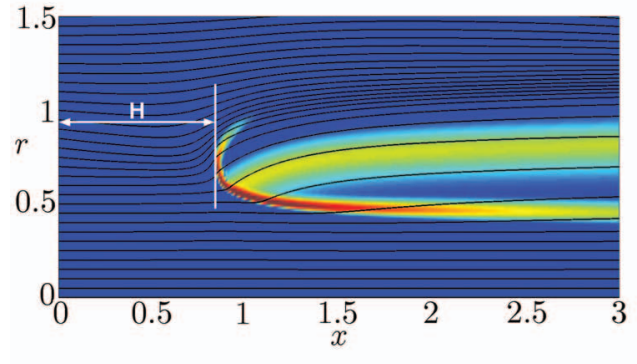

(a)

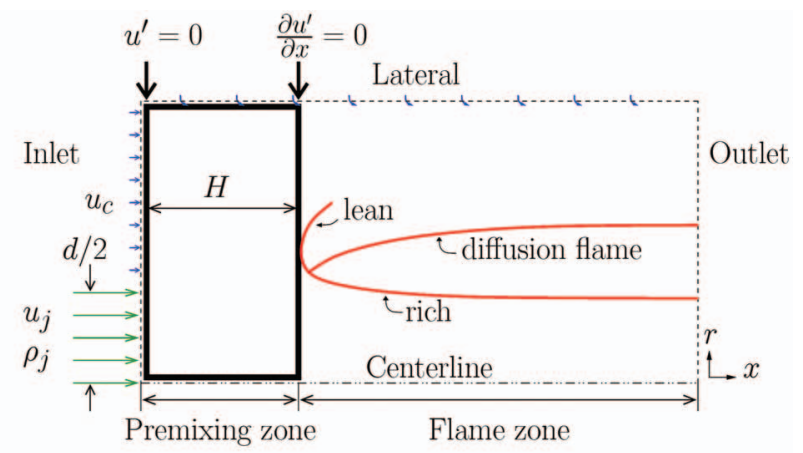

(b)

FIG. 1. (Color) (a) An axial cross section taken from DNS data of a steady lifted flame near the critical lift-off height. The flame is axisymmetric about the centerline along the bottom edge of the figure. Color contours of reaction rate are shown together with fluid streamlines (black). (b) A schematic view of the lifted flame. The stability analysis focuses on the region outlined by the heavy black lines, known as the premixing zone. In this region, the streamlines shown in (a) are nearly parallel.

\section{LIFTED FLAMES}

\section{A. Flow configuration}

Since a lifted flame on a round variable-density fuel jet serves as an example system throughout this paper, we briefly describe its configuration here. Figure 1(a) shows a cross section through a stable, axisymmetric lifted flame, obtained by direct numerical simulation (DNS). For the simulation, an identical formulation of governing equations was used as that described in Ref. 3. Namely, the low-Machnumber equations, ${ }^{14}$ together with a conserved scalar equation and a one-step Arrhenius reaction source term, were solved. The equations were advanced in time until the steady solution shown in Fig. 1(a) was obtained.

In this figure, the centerline is located along the bottom of the figure and a fuel jet enters through a nozzle of radius $d / 2=0.5$ from the left. The fuel-to-air density ratio $S$ $=\rho_{j} / \rho_{0}=0.143$ and Reynolds number $\operatorname{Re}=\rho_{j} u_{j} d / \mu=1000$, where $\rho_{j}, \rho_{0}, u_{j}, d$, and $\mu$ are the density of the jet, the density of the surrounding air, the jet velocity at the nozzle exit, the nozzle diameter, and the fluid viscosity, respectively. The jet profiles considered here are exactly the same as those used in Ref. 3, in brief, a version of Michalke's profile number $2,{ }^{15}$ with shear layer thickness $d /(2 \theta)=12.5$ where $\theta$ is the momentum thickness of the shear layer and $1 \%$ coflow so that $u_{c}=0.01 u_{j}$.

In Fig. 1(a), color contours of reaction rate reveal a triple flame structure at the flame base, similar to that observed in laboratory experiments and other numerical simulations. ${ }^{1,2,16,17}$ Important to the present investigation is that there exists an axially extended region of length $H$ (the lift-off height) where the fuel jet does not react. This region, outlined by the heavy black lines in Fig. 1(b), is bounded upstream by the nozzle and downstream by the lifted flame base and is known as the premixing zone since the pure fuel issuing from the nozzle may partially premix with surrounding air in this zone before encountering the flame. As shown by the fluid streamlines in Fig. 1(a), the flow in the premixing zone is nearly parallel and, for small enough density ratio $S$, absolutely unstable. ${ }^{18}$

\section{B. Dispersion relation}

Since the variable-density jet in the premixing zone is nearly parallel, we apply the locally parallel assumption to this region only and consider the dispersion relation of perturbations about the inlet profiles. Caution should be taken when applying the locally parallel assumption to viscous, and thus spatially developing, flows. In a previous study, ${ }^{13}$ however, it was shown that locally parallel stability analysis of a variable-density jet at the same Reynolds number ( Re $=1000)$ as considered in this paper was able to match the natural frequency observed from a fully nonlinear DNS to within 5\%. A subsequent study ${ }^{3}$ showed that the axial development of local stability characteristics in the premixing zone of a lifted flame followed closely the slow axial development of the same stability characteristics in a nonreacting variable-density jet, except in a small region in the immediate vicinity of the flame base. Therefore, we expect the locally parallel assumption to be valid over most of the premixing zone. The sudden change in stability properties encountered at the flame base, however, is modeled by enforcing a downstream boundary condition on the perturbations about the parallel flow in the premixing zone.

The perturbation dispersion relation for the parallel flow in the premixing zone is visualized in Fig. 2 by mapping grid points from the complex $\omega$ plane into the complex $k$ plane. $\omega_{r}$ varied over the interval $\left[\Delta \omega_{r}, 50 \Delta \omega_{r}\right]$ and $\omega_{i}$ varied over $\left[0,11 \Delta \omega_{i}\right]$ with the increments $\Delta \omega_{r}=0.0425$ and $\Delta \omega_{i}$ $=0.0449$ chosen so as to include the absolute frequency $\omega_{0}$. For each $\omega$, a spectrum of eigenvalues corresponding to spatial modes was solved by means of a spectral method described in Ref. 13. In the spectral method, the radial direction was discretized by 150 Chebychev modes in the case of the circles and 200 Chebychev modes in the case of the $\times$ s. A bilinear map was used to place the spectral collocation points on a stretched mesh, and a Dirichlet lateral boundary condition was implemented at $R_{\max }=20 \mathrm{~d}$, a distance sufficiently large so as to limit transverse confinement effects.

From our calculations, we find that modes with $k_{r}>0.15$ are well converged, i.e., they do not vary signifi- 


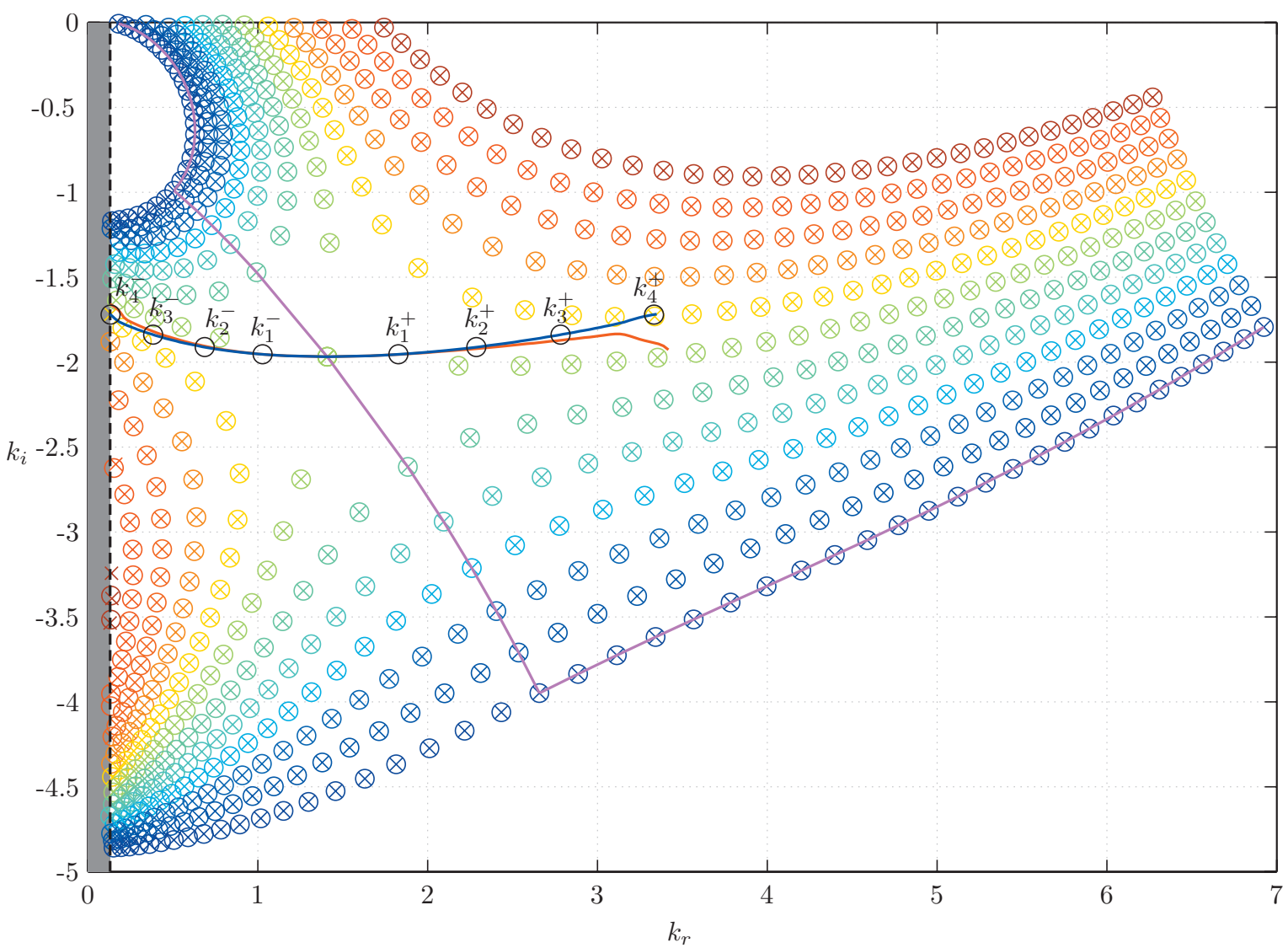

FIG. 2. (Color) Grid points of the complex $\omega$ plane are mapped through the dispersion relation of a round variable-density jet into the complex $k$ plane. The colors indicate values of $\omega_{i}$ of the original points, where red and blue correspond to high and low values, respectively. The magenta line represents the contour of steepest descent through the pinch point which then intersects the contour $\omega_{i}=0$. The black circles are an example of a discrete set of perfectly reflecting resonant modes which in general must lie on the blue solution curve. The red curve is the modification of this solution curve taking into account reflection coefficients.

cantly with resolution. On the other hand, convergence breaks down as the $k_{i}$ axis is approached (the gray region in Fig. 2). For an unconfined jet, a continuous spectrum exists along the $k_{i}$ axis, corresponding to modes which oscillate in the free stream. ${ }^{13}$ In the limit $r \rightarrow \infty$, the amplitude of the freestream oscillations diminishes as $\phi(r) \sim \sqrt{\pi /\left(2 k_{r} r\right)}$ $\times \exp \left(-k_{r} r\right){ }^{8,19}$ In the case of a confined jet (even a slightly confined jet such as the present case), the continuous spectrum is approximated by a series of $k^{-} / k^{-}$pinch points aligned close to the axis. ${ }^{10}$ As $R_{\max }$ increases, these pinch points move even closer to the axis and become more densely packed. In what follows, however, we consider only well-converged modes outside of the gray region. Note, however, that the width of the gray region may be reduced simply by increasing the resolution at the expense of increasing computation time.

The dispersion relation for the present locally parallel jet contains a pinch point at $k_{0}=1.409-1.968 i$ corresponding to a frequency $\omega_{0}=1.063+0.269 i$. Since $\operatorname{Im}\left(\omega_{0}\right)>0$, the flow is absolutely unstable. At the pinch point we measure $\omega_{k k}^{0}$ $=0.1681+0.0402 i$. The points in Fig. 2 were colored according to $\omega_{i}$ with blue and red corresponding to small and large values, respectively. The magenta curve thus follows a path of steepest descent through the pinch point before intersecting a contour of $\omega_{i}=0$ on either side of the pinch point. This curve forms a portion of the contour $F$ of the impulse response integral discussed in the next section. Finally, the blue curve forms the locus of resonant mode solutions as discussed below.

\section{LINEARIZED GINZBURG-LANDAU MODEL}

\section{A. Twisted pinch points}

A generic pinch point $\left(k_{0}, \omega_{0}\right)$ satisfies both the dispersion relation $D(k, \omega)=0$ and the zero group velocity criterion $\partial \omega / \partial k=0$. Therefore, the lowest order Taylor series expansion of the dispersion relation about the point $\left(k_{0}, \omega_{0}\right)$ is

$$
\omega-\omega_{0}=\frac{1}{2} \omega_{k k}^{0}\left(k-k_{0}\right)^{2} .
$$

Equation (1) describes a saddle in the complex $k$ plane where the quadratic term gives rise to two branches of solutions. When the instability is convective, the $F$ contour of the impulse response integral

$$
G(x, t)=\frac{1}{4 \pi^{2}} \int_{L} \int_{F} \frac{\exp (i k x-i \omega t)}{D(k, \omega)} d k d \omega
$$

may be deformed to pass through the point $\left(k_{0}, \omega_{0}\right)$ so that the $L$ contour taken as the real $\omega$ axis does not cross zeros of $D(k, \omega)$ (see Ref. 20 for details). Furthermore, the $F$ contour 
(a)

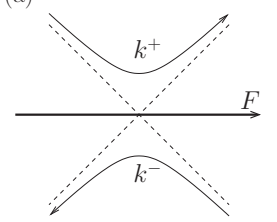

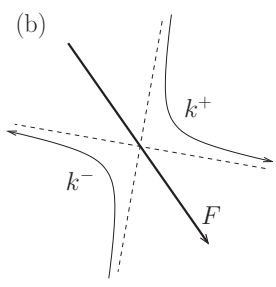

FIG. 3. (a) Normal pinch point with $\operatorname{Im}\left(\omega_{k k}^{0}\right)<0$. (b) Twisted pinch point for the case of the variable-density jet $\left[\operatorname{Im}\left(\omega_{k k}^{0}\right)>0\right]$.

must follow a path of steepest descent when $\omega_{i}>0$ in order to avoid oscillating terms in the integral. ${ }^{21}$

Figure 3 shows a schematic of the local behavior of the dispersion relation about a generic pinch point for two different values of $\omega_{k k}^{0}$. The curves represent contours of constant $\omega_{i}$ mapped into the complex $k$ plane through Eq. (1). Assuming $\omega_{i}^{0}>0$, the $F$ contour follows the path of steepest descent between these curves, separating the $k$ plane into two branches of upstream and downstream propagating modes, labeled $k^{-}$and $k^{+}$, respectively. The separating contour $F$ is an important feature of a pinch point since in general there may exist other saddle points satisfying the dispersion relation and the zero group velocity criterion but through which $F$ does not pass. In this case, these saddles are composed of the coalescence of two upstream propagating $k^{-}$modes or of two downstream propagating $k^{+}$modes and thus do not contribute to the integral. ${ }^{21}$

If the modulus of $\omega_{k k}^{0}$ determines the curvature of the saddle, its phase defines its orientation. Figure 3(a) depicts the orientation of the saddle for the LGL equation used in Ref. 4 with $D(k, \omega ; \mu)=U k+i\left(\mu-k^{2}\right)-\omega$. In this case, $\omega_{k k}$ $=-2 i$ and the $F$ contour reduces simply to the real $k$ axis. Figure 3(b) depicts the local behavior of the pinch point for the variable-density jet. In this case, the saddle is rotated a little more than $45^{\circ}$ in the clockwise direction. In general, if saddle 3 (a) is rotated more than $45^{\circ}$ in either direction, $\operatorname{Im}\left(\omega_{k k}^{0}\right)$ becomes positive, and the pinch point is said to be twisted.

Note that if the twisted saddle 3(b) was extended over the entire $k$ plane, i.e., if $\omega_{k k}^{0}$ were a constant as for the LGL model, the $F$ contour could not separate the $k^{+}$and $k^{-}$ branches since $F$ must start and end on the real $k$ axis. In other words, a LGL equation with $\operatorname{Im}\left(\omega_{k k}^{0}\right)>0$ would not satisfy causality ${ }^{22}$ since $G(x, t)$ would not be zero for $t<0$, the response then preceding the impulsive cause at $t=0$. It seems that we have arrived at a paradox because the dispersion relation of the physical variable-density jet appears to contain a pinch point associated with a noncausal LGL equation. This paradox is resolved, however, by considering resonant modes which incorporate the behavior of the dispersion relation farther away from the pinch point.

\section{B. Resonant modes}

In a convectively unstable system of finite length, as shown in Fig. 4, a downstream propagating wave $k^{+}$may be reflected into an upstream propagating wave $k^{-}$at the downstream boundary. The $k^{-}$wave may then be reflected back

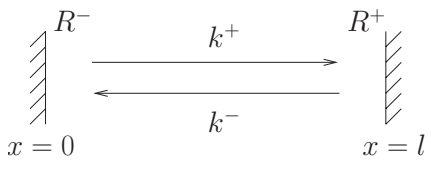

FIG. 4. Schematic adapted from Ref. 5 of a resonant mode composed of a downstream $k^{+}$wave superimposed with an upstream $k^{-}$wave coupled through reflections $R^{-}$and $R^{+}$at the upstream and downstream boundaries, respectively.

into the $k^{+}$wave at the upstream boundary, completing a cycle. If the frequency $\omega^{+}$associated with the $k^{+}$wave matches the frequency $\omega^{-}$of the $k^{-}$wave, then the waves will resonate. The superposition of the resonating waves forms a resonant mode with frequency $\omega_{G}=\omega^{+}=\omega^{-}$,

$$
f(x, t)=\left[\exp \left(i k^{+} x\right)-\exp \left(i k^{-} x\right)\right] \exp \left(-i \omega_{G} t\right) .
$$

Here, we have chosen the relative amplitudes of the two waves so that a Dirichlet boundary condition is satisfied at $x=0$ [i.e., $f(0, t)=0$ ]. In order to also satisfy a Dirichlet boundary condition at $x=l$, the wavenumbers $k^{+}$and $k^{-}$must satisfy $^{5}$

$$
\exp \left[i\left(k^{+}-k^{-}\right) l\right]=1 .
$$

This leads to the following conditions necessary for a resonant mode:

$$
\omega^{+}=\omega^{-}, \quad \operatorname{Re}\left(k^{+}-k^{-}\right)=\frac{2 n \pi}{l}, \quad \operatorname{Im}\left(k^{+}-k^{-}\right)=0,
$$

where $n$ is a nonzero integer. The third condition above is also known as the Kulikovskii criterion. ${ }^{23}$ The eigenfrequency of the resonant mode $\omega_{G}$ is in general complex. If its imaginary part $\omega_{G i}$ is larger than $\omega_{i}^{0}$, the $F$ contour corresponding to an $L$ contour with $\operatorname{Im}(\omega)=\omega_{i}^{0}$ passes through the saddle and separates spatial modes with frequencies corresponding to $\omega_{i}=\omega_{G i}$ into generalized $k^{-}$and $k^{+}$branches. For $\omega_{G i}$ lower than $\omega_{i}^{0}$, the resonance conditions are identical to Eq. (5), except that they are not restricted to $k^{+}$and $k^{-}$waves since they cannot be defined anymore. In this case, all couples of resonant waves are valid. This means that the addition of boundaries to a flow produces resonant modes with modified (real) frequencies and growth rates compared to the unbounded absolute mode. As we will see, in the case of twisted pinch points, the growth rate of resonant modes may be larger than that of the absolute mode.

Together with the dispersion relation, the first and third conditions of Eq. (5) describe two continuous curves through the four-dimensional space $\left(k_{r}, k_{i}, \omega_{r}, \omega_{i}\right)$ which meet at a saddle point $k_{0}$, as shown by the heavy black curves in Fig. 2. These two branches map through the dispersion relation onto a single curve in the complex $\omega$ plane, which ends at $\omega_{0}$. Along the continuous curve described by the first and third conditions, the second condition of Eq. (5) is satisfied only at discrete locations. This results in a discrete spectrum of resonant modes each corresponding to a different branch of the complex logarithm function implicit in Eq. (4).

As previously mentioned, a lifted flame may be modeled by a Neumann boundary condition $f^{\prime}(l, t)=0$ to the region of parallel flow just upstream. Taking the derivative of Eq. (3) 
gives the following condition for a resonant mode satisfying a Dirichlet upstream boundary and a Neumann downstream boundary:

$$
\exp \left[i\left(k^{+}-k^{-}\right) l\right]=\frac{k^{-}}{k^{+}}
$$

While similar to Eq. (4), this condition is transcendental and must be solved numerically. Like Eq. (4), however, it still yields a discrete spectrum of modes associated with the branches of the logarithm function. Note also that as $k^{-}$ $\rightarrow k^{+}$(when $l \rightarrow \infty$ ) the two conditions (4) and (6) become increasingly similar, as do the solutions. Therefore, since the behaviors of the two families of solutions are similar in the region near the pinch point, we choose to use condition (4) for simplicity.

We note here that condition (4) assumes perfect reflection at the upstream and downstream boundaries. This result may be generalized by introducing reflection coefficients $R^{-}$ and $R^{+}$at the upstream and downstream boundaries such that $\phi^{+}(0)=R^{-} \phi^{-}(0)$ and $\phi^{-}(l)=R^{+} \phi^{+}(l) . R^{-}$specifies how much of the upstream propagating wave is reflected into the downstream propagating wave at the upstream boundary. Likewise, $R^{+}$gives the amount of the downstream propagating wave that is reflected into the upstream propagating wave at the downstream boundary. Taking into account the reflection coefficients, the resonant condition becomes

$$
\exp \left[i\left(k^{+}-k^{-}\right) l\right]=\frac{1}{R^{+} R^{-}}
$$

In the case of perfect reflection with $R^{-}=R^{+}=-1$, this condition reduces to the previous result in Eq. (4).

Condition (7) may be rearranged as

$$
\left(k_{i}^{+}-k_{i}^{-}\right)=\frac{\operatorname{Re}\left[\ln \left(R^{+} R^{-}\right)\right]}{2 \pi n-\operatorname{Im}\left[\ln \left(R^{+} R^{-}\right)\right]}\left(k_{r}^{+}-k_{r}^{-}\right),
$$

where $n$ is the discrete mode number, and we have eliminated $l$. To study stabilization, we consider only $n=1$, since this is the last mode to disappear, as we shall see.

\section{Resonant modes near a pinch point}

As discussed in the previous section, the discrete spectrum of resonant modes lies along a continuous curve, the discrete locations depending on the domain size $l$. As $l \rightarrow \infty$, the discrete spectrum becomes increasingly dense along the curve, although when $l=\infty$, we may only observe the absolute mode at $\left(k_{0}, \omega_{0}\right)$ with zero group velocity. Since the two branches of the locus of solutions meet at the pinch point $\left(k_{0}, \omega_{0}\right)$, we consider its behavior in the immediate vicinity of a generic pinch point described to lowest order by the dispersion relation (1). If we apply the first condition of Eq. (5) to this dispersion relation, we find that

$$
\left(k^{+}-k_{0}\right)^{2}=\left(k^{-}-k_{0}\right)^{2} .
$$

This can be expressed in terms of its real and imaginary parts,

$$
k_{r}^{+}\left(k_{r}^{+}-2 k_{r}^{0}\right)=k_{r}^{-}\left(k_{r}^{-}-2 k_{r}^{0}\right),
$$

(a)

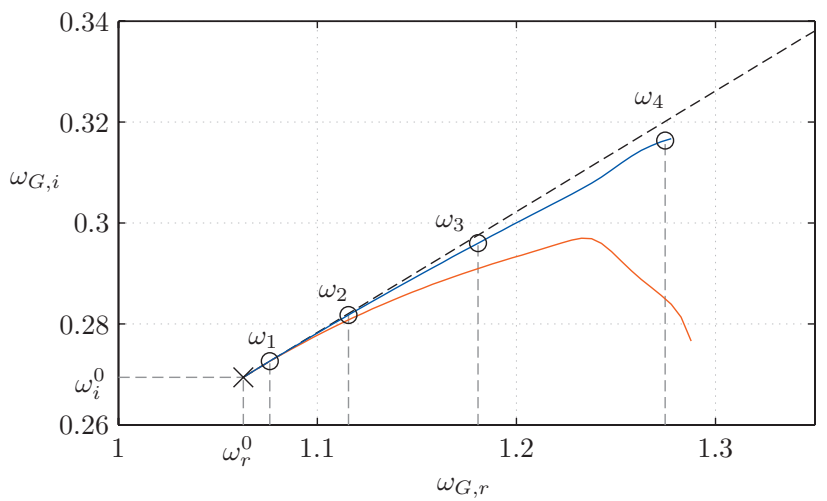

(b)

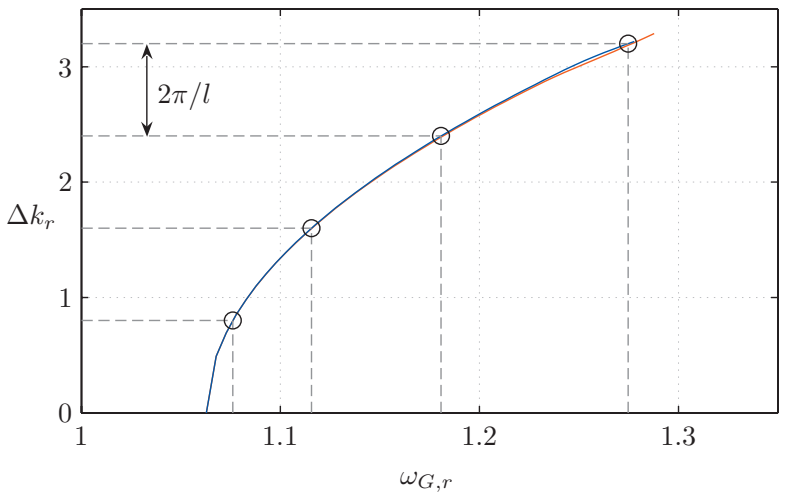

FIG. 5. (Color) (a) Resonant modes in the complex $\omega$ plane. The three curves represent the locus of solutions computed from the LGL model (black dashed line), the full dispersion relation assuming perfect reflection (blue), and the full dispersion relation using the overlap integral to find reflection coefficients (red). All three curves begin at the absolute frequency shown by the $\times$. Circles $\omega_{1}, \ldots, \omega_{4}$ are a discrete set of perfectly reflecting resonant modes, corresponding to those shown in Fig. 2. (b) Resonant modes in the $\left(\omega_{r}, \Delta k_{r}\right)$ plane. The discrete modes (circles) are equidistant in the vertical direction. This determines the spacing along the $\omega_{r}$ axis, which is then used in (a).

$$
k_{r}^{+}\left(k_{i}^{+}-k_{i}^{0}\right)=k_{r}^{-}\left(k_{i}^{-}-k_{i}^{0}\right) .
$$

From the second equation, assuming $k_{r}^{+} \neq k_{r}^{-}$and noting that $\left(k_{i}^{+}-k_{i}^{0}\right)=\left(k_{i}^{-}-k_{i}^{0}\right)$, we find

$$
k_{i}^{+}=k_{i}^{-}=k_{i}^{0},
$$

which means that in the complex $k$ plane, all of the resonant modes appear on the horizontal $\operatorname{line} \operatorname{Im}(k)=k_{i}^{0}$. Note that this condition does not depend on $\omega_{k k}^{0}$ so no matter how the pinch point is twisted, the resonant modes always align on this horizontal line. $\omega_{k k}^{0}$ does affect the locus of solutions in the $\omega$ plane, however. Since the factor $\left(k-k_{0}\right)^{2}$ on the right hand side of Eq. (1) is always real and positive along the line $\operatorname{Im}(k)=k_{i}^{0}$, the two branches of the locus of solutions in the complex $k$ plane map onto a single line beginning at $\omega_{0}$ in the complex $\omega$ plane, as shown by the sloping dashed line in Fig. 5(a). The orientation of this line is given simply by the argument of $\omega_{k k}^{0}$.

If the pinch point is twisted, $\operatorname{Im}\left(\omega_{k k}^{0}\right)>0$ and the solution curve is directed into the upper half $\omega$ plane. Supposing that dispersion relation (1) was valid over the entire $\omega$ plane, this would lead to a sequence of resonant modes with increas- 
ingly strong growth rates. A Laplace contour integral, closed in the upper half-plane, would always contain at least one resonant mode in this case, violating causality (as already noted). Therefore, we retain the paradox introduced in Sec. III A up to this point. In the next section, however, we resolve this paradox by considering the behavior of resonating modes under the full dispersion relation and show that the LGL model does indeed give the correct behavior close to the pinch point.

\section{FULL DISPERSION RELATION}

To find resonant modes under the full dispersion relation satisfying Eq. (4) we apply the following numerical procedure. Starting at the absolute frequency $\omega_{0}$, we gradually increase $\omega_{r}$. For each $\omega_{r}$ above $\omega_{0, r}$, a Newton iteration is used to adjust $\omega_{i}$ so that $k_{i}^{+}=k_{i}^{-}$. This defines a curve along which the first and third conditions of Eq. (5) are satisfied, as shown by the blue curve in Fig. 2. As predicted by the analysis of the previous section, this curve passes horizontally through the pinch point. Once it is sufficiently far from the pinch point, however, it curves to take into account the overall shape of the full dispersion relation. The remaining discrete condition of Eq. (5) may then be satisfied along this curve, defining a discrete set of resonating modes for a given system length $l$. Shown by the black circles in Fig. 2, this discrete set is divided by the magenta line into downstream propagating modes $k_{1}^{+}, \ldots, k_{4}^{+}$and upstream propagating modes $k_{1}^{-}, \ldots, k_{4}^{-}$.

The two branches of solutions in the complex $k$ plane map through the dispersion relation onto a single curve in the complex $\omega$ plane, shown in blue in Fig. 5(a). In the complex $\omega$ plane, this solution curve begins at $\omega_{0}$ and is initially oriented in the direction $\arg \left(\omega_{k k}^{0}\right)$ represented by the sloped dashed line. In this case, the initial direction is toward the upper half-plane since $\operatorname{Im}\left(\omega_{k k}^{0}\right)>0$. This means that there exists a range of resonant modes with growth rates larger than that of the absolute mode.

In order to satisfy the remaining, discrete condition of Eq. (5), we first draw the solution curve in the $\left(\omega_{r}, \Delta k_{r}\right)$ plane as shown in Fig. 5(b), where $\Delta k_{r}=\operatorname{Re}\left(k^{+}-k^{-}\right)$may be understood graphically in Fig. 2 as the horizontal separation between two resonating modes on either side of the magenta line. At the pinch point, $\Delta k_{r}=0$ and then increases as $\omega_{r}$ is increased and the modes move away from the pinch point. In Fig. 5(b), the discrete condition is satisfied along regularly spaced horizontal lines where $\Delta k_{r}=2 n \pi / l$. These horizontal lines intersect the curve shown in Fig. 5(b) at the circles, where we have taken $2 \pi / l=0.8$ for illustration. These points define a discrete spectrum of $\omega_{r}$ values at which the discrete condition is satisfied. Note that the slope of curve in the $\left(\omega_{r}, \Delta k_{r}\right)$ plane determines the spacing of the $\omega_{r}$ values, which cluster near $\omega_{r}^{0}$ since the slope of the curve is steepest at this point. The $\omega_{i}$ values corresponding to this spectrum are shown by the circles in Fig. 5(a).
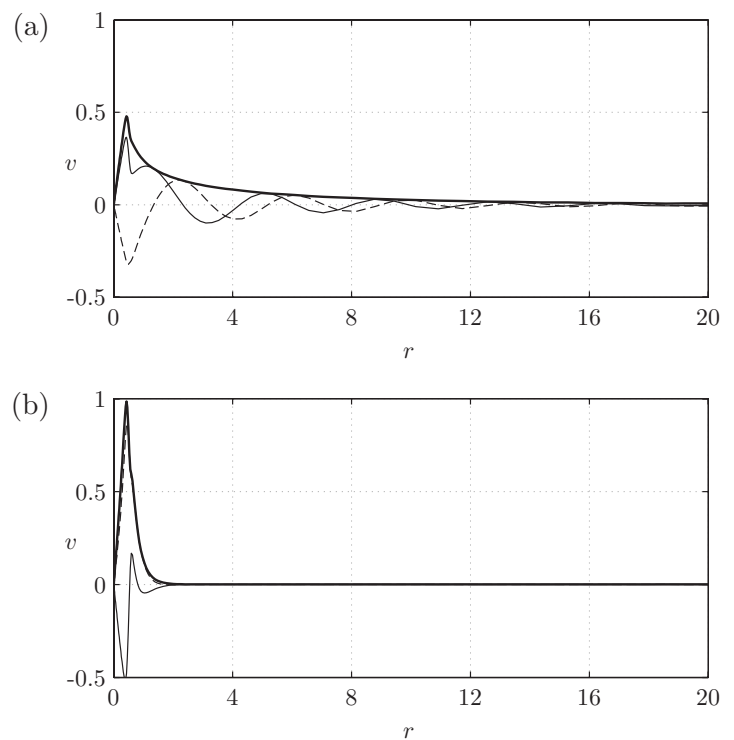

FIG. 6. Normalized radial profiles of the resonating (a) $k^{-}$and (b) $k^{+}$waves. The modulus (heavy solid line), real (thin solid line), and imaginary (thin dashed line) parts of the radial velocity $v$ are displayed. This example was taken for $k^{-}$approaching the imaginary $k$ axis, so that the difference of radial attenuation between the two modes is apparent. In this case, the total reflection was measured to be $\left|R^{+} R^{-}\right|=0.7$.

\section{DISCUSSION}

As $l$ decreases, $2 \pi / l$ increases and the spacing of the discrete resonant modes along the solution curve increases. For small enough $l$, the spacing $2 \pi / l$ between horizontal lines in Fig. 5(b) is large enough so that none of them intersects the solution curve. In this case, no unstable resonant mode exists and the system is rendered stable. This restabilization of the flow with strong streamwise confinement agrees with results of numerical simulations of a lifted flame. In the simulations, lifted flames with sufficiently low lift-off heights were observed to be stable while their counterparts with greater lift-off heights supported self-sustaining instabilities. $^{3}$

In Fig. 5(a), the termination of the solution curve at a finite growth rate is somewhat perplexing. After all, the location of this end point was fixed by a numerical convergence criterion, and thus should not be related to the underlying physics. This artifact can be explained, however, by considering reflection coefficients at the upstream and downstream boundaries. Up to this point, we have assumed perfect reflection of the $k^{+}$and $k^{-}$waves. Near the end point of the solution curve, this assumption breaks down. Recall that the radial attenuation of the eigenmode associated with each wave depends on the axial wavenumber $k_{r}$ as $\phi(r)$ $\sim \sqrt{\pi /\left(2 k_{r} r\right)} \exp \left(-k_{r} r\right)$. Figure 6 shows the radial attenuation for the $k^{-}$and $k^{+}$waves, taken near the end point of the solution curve. At this point, $k_{r}^{-}$is small so most of the energy of the $k^{-}$wave is contained in the free stream. At the same time, $k_{r}^{+}$is large, which means that most of the energy of the downstream wave is concentrated near the jet core. In this case, we expect the radial separation of energy carrying regions to yield poor reflection coefficients, so that we must 
use condition (7) when solving for resonant modes instead of Eq. (4).

To quantify this effect, we resort to the overlap integral taken from the theory of optics. ${ }^{24}$ The downstream reflection coefficient $R^{+}$is given by

$$
R^{+}=-\frac{\int \phi^{+}(r)\left[\phi^{-}(r)\right]^{*} r d r}{\int \phi^{+}(r)\left[\phi^{+}(r)\right]^{*} r d r}
$$

where the $*$ denotes the adjoint. A similar formula holds for $R^{-}$, with all of the + and - superscripts exchanged. In the numerator, we take the adjoint with respect to the basis formed by all upstream propagating $\phi^{-}$waves, whereas in the denominator, the adjoint is formed with respect to the basis formed by all downstream propagating $\phi^{+}$waves. These two different adjoint operators are a consequence of the fact that while we match the values of the state variables at the boundaries, we do not specify their axial derivatives. Therefore, only one-half of the state information is used in forming each boundary condition. Simply put, the reflection coefficient $R^{+}$is found by projecting the normalized resonant wave $\phi^{+}$onto the basis of all upstream propagating waves and then finding the component of the projection in the direction corresponding to the upstream propagating resonant wave. $R^{-}$is derived in a similar manner. Note that the reflection coefficients derived from the overlap integral method may in general be complex, taking into account a possible phase shift between the upstream and downstream propagating waves. Also, since the system considered at present is non-normal, the modulus of the reflection coefficients may be greater than unity.

The red curves of Figs. 2 and 5 represent the modified solution curve using the overlap integral method to calculate reflection coefficients. The numerical method proceeds in much the same manner as before: for each $\omega_{r}$, we search for an $\omega_{i}$ such that Eq. (7) is satisfied. Note, however, that this means that new sets of adjoint modes must be computed at each step in addition to the direct modes, since the reflection coefficients depend on $\omega_{i}$. Numerically, this is accomplished using a linear least-squares algorithm to find the optimal projection of the resonant $\phi^{+}$wave onto a basis of $\phi^{-}$waves and vice versa.

Near the pinch point, the modified solution curve follows the original solution curve. Since $\left|R^{+} R^{-}\right| \approx 1$ in this region, conditions (4) and (7) are nearly equivalent. As the $k^{-}$ resonant wave approaches the imaginary axis, however, the reflection coefficient decreases rapidly and the two solution curves diverge. This corresponds to a sharp damping of the resonant mode as shown by the red curve in Fig. 5(a). Therefore, we interpret the disappearance of perfectly reflecting resonant modes past the end of the blue curve to be a model for the rapid stabilization of these modes owing to the deterioration of the reflection coefficients as the imaginary $k$ axis is approached.

We end our discussion by noting that when $l$ is large enough, two or more resonant modes are predicted with growth rates larger than that of the absolute mode. Furthermore, these modes occur at slightly different frequencies and thus may interact nonlinearly via wave triad interactions, ${ }^{25}$ forcing harmonics at multiples of the frequency differences. This suggests that the low-frequency component observed in the simulations of lifted flames may arise from a beating between two resonant modes with closely spaced frequencies.

\section{CONCLUSIONS}

In this paper, we have investigated the behavior of resonant modes arising from streamwise confinement. In particular, we have demonstrated that systems containing twisted pinch points with $\operatorname{Im}\left(\omega_{k k}^{0}\right)>0$, such as the variable-density jet, may be destabilized by streamwise confinement, contrary to previous results. ${ }^{4,5}$ The source of this destabilization may be understood by modeling the dispersion relation at the pinch point by a LGL equation. Although the resulting LGL equation with $\operatorname{Im}\left(\omega_{k k}^{0}\right)>0$ is noncausal, we have shown that it still predicts the correct behavior of resonant modes near the pinch point. This has been demonstrated by applying the general resonance condition (4) to both the LGL model equation and the full dispersion relation, the results of which are shown in Fig. 5(a) by the black dashed and blue lines, respectively. Near the absolute frequency, the blue curve converges to that predicted by the LGL equation. Furthermore, since this line is oriented toward the upper half-plane there exists a range of frequencies $\omega_{r}$ in which resonant modes have larger growth rates $\omega_{i}$ than that of the absolute mode $\omega_{i}^{0}$.

While this type of destabilization is of interest to physical systems such as the lifted flame, it may also be an important consideration for numerical simulations. In a simulation of a system containing a twisted pinch point, reflections from inflow and outflow numerical boundary conditions would in this case lead to oscillations at a frequency corresponding to the resonant mode with largest $\omega_{i}$, perhaps overshadowing the desired absolute mode. Note that increasing the system length $l$ would actually make the situation worse in this case, since the solution curve would become increasingly populated with unstable resonant modes.

The upward trend of the solution curves shown in Fig. 5 cannot continue indefinitely, however. If this were the case, we could not find a contour $L$ closing the integral (2) in the upper half-plane. Instead, the paradox of the noncausality of the LGL equation is resolved by considering that it is only a model valid close to the pinch point. Taking into account the overall shape of the full dispersion relation reveals a different behavior of resonant modes far away from the pinch point. In the case of perfectly reflecting boundaries, the blue solution curve appears to terminate at a finite growth rate. When reflection coefficients are considered, this termination is replaced by a rapid damping of the resonant modes as shown by the red curve in Fig. 5. This damping is caused by a deterioration of the reflection coefficients as the imaginary $k$ axis is approached. In either case, this leads to an eventual stabilization as the system length $l$ is decreased. Just before stabilization, however, the remaining resonant mode has the strongest growth rate. Therefore, the final stabilization for short $l$ may be viewed as a sudden, catastrophic transition. In summary, for large $l$, the system is destabilized by resonant 
modes with growth rates larger than the absolute growth rate. As $l$ decreases, the destabilization becomes stronger until no resonant modes remain along the solution curve, at which point the system is rendered catastrophically stable.

The theory discussed in this paper agrees with results of DNS of lifted flames on variable-density jets. ${ }^{3}$ In the simulations, a critical lift-off height was found, below which the system was rendered stable. Although the linear growth rates were not obtained from the nonlinear simulations, the frequency of the instability was observed to increase as the lift-off height was decreased toward this critical lift-off height. This agrees well with the results presented in Fig. 5, where $\omega_{r}$ of all resonant modes increases as $l$ is decreased. Finally, because there may be more than one resonant mode present along the solution curve, each with a slightly different frequency, this suggests that the low-frequency fluctuation observed in the DNS may be caused by a beating between two different modes. Near the critical lift-off height, however, we suspect that strong nonlinear effects may also play a role in sustaining this low-frequency phenomenon, since our model predicts an extremely rapid transition between stability and instability at this point.

\section{ACKNOWLEDGMENTS}

This research has been supported by the ANR Chaires d'Excellence program.

${ }^{1}$ S. Ghosal and L. Vervisch, "Theoretical and numerical study of a symmetrical triple flame using the parabolic flame path approximation," J. Fluid Mech. 415, 227 (2000).

${ }^{2}$ A. Linán, E. Fernández-Tarrazo, M. Vera, and A. L. Sánchez, "Lifted laminar jet diffusion flames," Combust. Sci. Technol. 177, 933 (2005).

${ }^{3}$ J. W. Nichols and P. J. Schmid, "The effect of a lifted flame on the stability of round fuel jets," J. Fluid Mech. 609, 275 (2008).

${ }^{4}$ J.-M. Chomaz and A. Couairon, "Against the wind," Phys. Fluids 11, 2977 (1999).

${ }^{5} \mathrm{O}$. Doaré and E. de Langre, "The role of boundary conditions in the instability of one-dimensional systems," Eur. J. Mech. B/Fluids 25, 948 (2006).

${ }^{6}$ M. P. Juniper and S. M. Candel, "The stability of ducted compound flows and consequences for the geometry of coaxial injectors," J. Fluid Mech. 482, 257 (2003).

${ }^{7}$ J. J. Healey, "Enhancing the absolute instability of a boundary layer by adding a far-away plate," J. Fluid Mech. 579, 29 (2007).

${ }^{8}$ M. P. Juniper, "The effect of confinement on the stability of non-swirling round jet/wake flows," J. Fluid Mech. 605, 227 (2008).

${ }^{9}$ J. J. Healey, "A new convective instability of the rotating-disk boundary layer with growth normal to the disk," J. Fluid Mech. 560, 279 (2006).

${ }^{10} \mathrm{M}$. P. Juniper, "The full impulse response of two-dimensional jet/wake flows and implications for confinement," J. Fluid Mech. 590, 163 (2007).

${ }^{11}$ D. G. Crighton, "The 1988 Rayleigh medal lecture: Fluid loading-the interaction between sound and vibration," J. Sound Vib. 133, 1 (1989).

${ }^{12}$ R. A. W. Haddon, "Exact evaluation of the response of a layered elastic medium to an explosive point source using leaking modes," Bull. Seismol. Soc. Am. 76, 1755 (1986).

${ }^{13}$ J. W. Nichols, P. J. Schmid, and J. J. Riley, "Self-sustained oscillations in variable-density round jets," J. Fluid Mech. 582, 341 (2007).

${ }^{14}$ P. A. McMurtry, W. H. Jou, J. J. Riley, and R. W. Metcalfe, "Direct numerical simulations of mixing layers with heat release," AIAA J. 24, 962 (1986).

${ }^{15}$ A. Michalke, "Survey on jet instability theory," Prog. Aerosp. Sci. 21, 159 (1984).

${ }^{16}$ A. Joedicke, N. Peters, and M. Mansour, "The stabilization mechanism and structure of turbulent hydrocarbon lifted flames," Proc. Combust. Inst. 30, 901 (2005).

${ }^{17}$ S. H. Won, J. Kim, K. J. Hong, M. S. Cha, and S. H. Chung, "Stabilization mechanism of lifted flame edge in the near field of coflow jets for diluted methane," Proc. Combust. Inst. 30, 339 (2005).

${ }^{18}$ P. A. Monkewitz and K. D. Sohn, "Absolute instability in hot jets," AIAA J. 26, 911 (1988).

${ }^{19}$ J. W. Nichols, 2005, "Simulation and stability analysis of jet diffusion flames," Ph.D. thesis, University of Washington.

${ }^{20}$ R. J. Briggs, Electron-Stream Interaction with Plasmas (MIT, Cambridge, 1964).

${ }^{21}$ M. P. Juniper, "The effect of confinement on the stability of twodimensional shear flows," J. Fluid Mech. 565, 171 (2006).

${ }^{22}$ J.-M. Chomaz, P. Huerre, and L. G. Redekopp, "A frequency selection criterion in spatially developing flows," Stud. Appl. Math. 84, 119 (1991).

${ }^{23}$ A. G. Kulikovskii, cited in L. Landau and E. M. Lifshitz, Physical Kinetics, Course of Theoretical Physics, Vol. 10 (Pergamon, Oxford, 1981), p. 281.

${ }^{24}$ D. I. Babić, Y. Chung, N. Dagli, and J. E. Bowers, "Modal reflection of quarter-wave mirrors in vertical-cavity lasers," IEEE J. Quantum Electron. 29, 1950 (1993).

${ }^{25}$ P. J. Schmid and D. S. Henningson, Stability and Transition in Shear Flows (Springer-Verlag, New York, NY, 2001). 